\title{
RECOMMENDATIONS
}

OF

\section{BULGARIAN SOCIETY OF RHEUMATOLOGY FOR VACCINATION OF PATIENTS WITH MUSCULOSKELETAL AND AUTOIMMUNE RHEUMATIC DISEASES}

\author{
Work group: \\ Prof. Rumen Stoilov, MD, PhD¹, Prof. Anastas Batalov, MD, PhD, Assoc. Prof. Mariana Ivanova, MD, PhD ${ }^{1}$, \\ Nikolay Stoilov, MD, PhD1, Vladimira Boyadhzieva, MD, PhD', Prof. Dobroslav Kyurkchiev, MD, PhD² \\ ${ }^{1}$ Rheumatology Department, UMHAT „Sv. Ivan Rilski“, MF, MU - Sofia \\ ${ }^{2}$ Laboratory of Clinical Immunology, UMHAT „Sv. Ivan Rilski“, MF, MU - Sofia \\ ${ }^{3}$ Rheumatology Clinic, UMHAT „Kaspela“, MF, MU - Plovdiv
}

The COVID-19 pandemic is an important global cause of morbidity, mortality and pressure on countries' health systems. Data from the EULAR COVID-19 register as of 1 December 2020 showed that approximately 3590 patients with rheumatic diseases developed SARS-CoV-2.

Several high-quality studies from this period indicate that the risk of COVID-19 infection and the course of treatment in patients with the most common autoimmune rheumatic diseases is similar to the general population. Only 2 months later, data from the EULAR register show that as of 1 February 2021, 4934 patients with rheumatic diseases had contracted SARS-CoV-2. Of these, 1911 (38.7\%) were hospitalized and 402 (8.14\%) died. The results from the COVID-19 Global Rheumatology Alliance (GRA) register are similar for the United States. 5751 patients with COVID-19 were registered, $1837(31.89 \%)$ of whom were hospitalized and 347 $(6.02 \%)$ died. This means that mortality in patients with autoimmune rheumatic diseases is 2-3 times higher than in the general population.

New data from the GRA registry reported in the Annals of the Rheumatic Diseases reveal that the risk of death from COVID-19 is $87 \%$ higher in patients with moderate to high disease activity than in those who achieved remission or low disease activity. For this reason, the Bulgarian Society of Rheumatology, at the end of last year, recommended to rheumatologists and patients, in the conditions of COVID-19 pandemic, not to delay the inclusion of conventional, biological or targeted synthetic disease-modifying antirheumatic drugs, unless a justified reason (intolerance, exhaustion, infectious or other disease incompatible with these drugs) is present.

The growing evidence that infection with COVID-19 in patients with autoimmune rheumatic diseases can have serious health consequences gave the Bulgarian Society of Rheumatology reason to discuss and propose recommendations aimed at preserving the health and life of this group of patients.

\section{SUMMARY OF EVIDENCE AND JUSTIFICATION}

- There is currently insufficient evidence that immunosuppression is an independent risk factor for the more severe course of COVID-19 infection in rheumatic patients. The continuous increase in the number of vaccinated immunocompromised individuals will allow for more solid future scientific conclusions.

- To date, data on vaccination against COVID-19 in patients who are immunosuppressed or suffer from autoimmune disease are very limited. Participants in the Pfizer-BioNTech vaccine trial were only non-immunosuppressed individuals, such as those with stable human immunodeficiency virus (HIV) infection, hepatitis $\mathrm{C}$ virus (HCV) or hepatitis $B$ virus (HBV). They did not receive immunosuppressive therapy during the study.

- People living with HIV who are considered immunocompetent can be vaccinated.

- The Summary of Product Characteristics for the Pfizer-BioNTech vaccine against COVID-19 states that: "Immunocompromised individuals, including those receiving immunosuppressant therapy, can have a lowered immune response to the vaccine".

- In general, non-replicating vaccines can be administered to immunocompromised individuals because the antigens in the vaccine cannot multiply. However, the frequency and duration of vaccine-induced immunity may be reduced. It is currently unknown whether immunocompromised individuals 
will be able to elicit a sufficient immune response to mRNA vaccines.

- People who are immunocompromised, either due to the underlying disease or immunosuppressive agents, are usually more susceptible to infections and severe illness after infection. The relative degree of immunodeficiency varies depending on the underlying disease, the progression of the disease and the medicinal product used, which suppresses immune function. Therefore, a balance of benefits and risks must be struck on a case-by-case basis.

- It is known that immunosuppressive therapy does not significantly alter the immune response after immunization with hitherto administered vaccines. There are insufficient data for the COVID-19 vaccine.

- The post-registration data obtained so far show that, given the severity of COVID-19 infection, the benefits of vaccination outweigh the potential risk of exacerbation or exacerbation of rheumatic disease.

Based on the scientific publications so far and the experience of the leading rheumatological associations (American College of Rheumatology, European League for Rheumatism, Canadian Rheumatological Association, British Rheumatology Association, Australian Rheumatology Association, etc.) the Bulgarian Society of Rheumatology recommends:

1. Patients with musculoskeletal and autoimmune rheumatic diseases may be vaccinated with inanimate vaccines, such as the vaccines against COVID-19 registered to date.

2. Vaccination is best done when the disease is in remission or with low disease activity. In the case of a life-threatening disease, vaccination against COVID-19 should be carried out as soon as possible.

3 . There is no preference for any of the vaccines against COVID-19 registered so far. Therefore, patients with autoimmune rheumatic diseases should receive the available vaccine.

4. Recommendations for immunosuppressive treatment before and after vaccination against COVID-19:

A) Medicinal products that do not change the dosage regimen and vaccination can be performed at any time after their reception: hydroxychloroquine; IVIG; glucocorticoids, prednisone equivalent < 20 mg/day; sulfasalazine; leflunomide; mycophenolate mofetil; azathioprine; cyclophosphamide (oral); TNFi; anti-IL-6R; anti-IL-1, anti-IL-17; anti-IL-12/23; anti-IL-23; belimumab; cyclosporine (orally).

B) Medicinal products, the dosage regimen of which is changed and vaccination is carried out at a specific time of their application:
- methotrexate: skipped 1 week after each dose of vaccine;

- JAK inhibitors (tofacitinib, baricitinib, upadacitinib): skipped 1 week after each dose of vaccine;

- abatacept, injectable: missed one week before and one week after the first dose of vaccine;

- abatacept, IV form: the vaccine is given 4 weeks after the last infusion, then skipped for one week and the next infusion of abatacept is given;

- rituximab: the COVID-19 vaccine is given approximately 4 weeks before the next infusion, the next rituximab infusion is delayed for 2-4 weeks after the second dose of vaccine - if possible;

- cyclophosphamide infusion: administered one week after each dose of COVID-19 vaccine.

5. Following vaccination against COVID-19, patients with rheumatic diseases should continue to follow all public health guidelines regarding physical distancing and other preventive measures.

6. We strongly recommend active monitoring of all vaccinated patients for potential adverse outcomes.

7. The immunization is carried out according to the plan and recommendations of the Ministry of Health and the National Vaccination Headquarters.

8. These recommendations will be updated as more information becomes available.

\section{Библиография / References}

1. Baker D, Roberts CAK, Pryce $G$ et al. COVID-19 vaccine-readiness for anti-CD20-depleting therapy in autoimmune diseases. Clin Exp Immunol 2020; 202: 149-61.

2. Benucci $M$, Infantimo $M$, Marotto $D$ et al. Vaccination against SARS-CoV-2 in patients with rheumatic diseases: doubts and perspectives. Clin Exp Rheumatol 2021; 39: 196-202

3. Calina D, Docea AO, Petrakis D et al. Towards effective COVID-19 vaccines: updates, perspectives and challenges (Review). Int J Mol Med 2020; 46: 3-16

4. Campochiaro C,Trignani G, Tomelleri A et al. Potential acceptance of COVID-19 vaccine in rheumatological patients: a monocentric comparative survey. Ann Rheum Dis: first published as 10.1136/annrheumdis-2020-219811 on 28 January 2021. DOI: http://dx.doi.org/10.1136/annrheumdis-2020-219811

5. Canadian Rheumatology Association Position Statement on COVID-19 Vaccination, Published on December 31, 2020.

6. COVID-19 Vaccine Clinical Guidance Summary for Patients with Rheumatic and Musculoskeletal Diseases Developed by the ACR COVID-19 Vaccine Clinical Guidance Task Force, 8 Feb 2021

7. European League Against Rheumatism (EULAR) (2020) EULAR-COVID-19 Database. Available from https ://www.eular .org/eular covid 19_datab ase.cfm. Accessed 19 Dec 2020

8. Ferro F, Elefante E, Puxeddu I et al. COVID-19: the new challenge for rheumatologists. First update. Clin Exp Rheumatol 2020; 38: 373-82. 
9. Furer V, Christien Rondaan, Marloes W Heijstek at all, 2019 update of EULAR recommendations for vaccination in adult patients with autoimmune inflammatory rheumatic diseases. Ann Rheum Dis. 2020;79(1):39-52. DOI: 10.1136/annrheumdis-2019-215882

10. Graham BS: Rapid COVID-19 vaccine development. Science 2020; 368: 945-6.

11. Grigoryan L, Pulendran B. The immunology of SARS-CoV-2 infections and vaccines. Semin Immunol 2020; 50: 101422.

12. Jackson LA, Anderson EJ, Rouphael NG et al. An mRNA Vaccine against SARSCoV-2 - Preliminary report. N Engl J Med 2020; 383: 1920-31.

13. Infantino M, damiani A, LI Gobbi F et al. Serological assays for SARS-CoV-2 infectious disease: benefits, limitations and perspectives. Isr Med Assoc J 2020; 22: 203-10.

14. Kipps S, Paul A, Vasireddy S (2020) Incidence of COVID-19 in patients with rheumatic disease: is prior health education more important than shielding advice during the pandemic? Clin Rheumatol 11:1-5.

15. Lurie N, Saville M, Hatchett $R$ et al. Developing Covid-19 Vaccines at Pandemic Speed. N Engl J Med 2020; 382:19691973 DOI: 10.1056/NEJMp2005630

16. Mehta P, Sanchez E, Moraitis E. at all. Influenza vaccination and interruption of methotrexate in adult patients in the COVID-19 era: an ongoing dilemmalnfluenza vaccination and interruption of methotrexate in adult patients in the COVID-19 era: an ongoing dilemma Pub-

Постъпил за печат: 01.03.2021 2.
lished:November 10, 2020 DOI:https://doi.org/10.1016/ S26659913(20)303921

17. Mercado NB, Zahn R, Wegmann F et al. Single-shot Ad26 vaccine protects against SARS-CoV-2 in rhesus macaques. Nature 2020; 586: 583-8.

18. Serling-Boyd N, D'Silva KM, Hsu TY, Wallwork R, Fu X, Gravallese EM et al (2020) Coronavirus disease 2019 outcomes among patients with rheumatic diseases 6 months into the pandemic. Ann Rheum Dis

19. Stoilov R, V. Boyadzhieva, N. Stoilov, M. Ivanova, A. Batalov, ZI. Kolarov, S. Monov, I. Sheytanov. Bulgarian Rheumatology Society recommendations for behavior during an epidemic with COVID-19. Rheumatology, 2020, 3; 3-6.

20. Strangfeld A, Schäfer M, Gianfrancesco MA, et al. Factors associated with COVID-19-related death in people with rheumatic diseases: results from the COVID-19 Global Rheumatology Alliance physician-reported registry. Annals of the Rheumatic Diseases. 27 January 2021. doi: 10.1136/annrheumdis-2020-219498

21. Tam S, Tanaka $Y$, Handa $R$ et al. Care for patients with rheumatic diseases during COVID-19 pandemic: A position statement from APLAR. Int J Rheum Dis. 2020;00:1-6. DOI: 10.1111/1756-185X.13863

22. Zhu F-C, Li Y-H, Guan X-H et al. Safety, tolerability, and immunogenicity of a recombinant adenovirus type- 5 vectored COVID-19 vaccine: a dose-escalation, open-label, nonrandomised, first-in-human trial. Lancet 2020; 395: 1845-54.

Submitted: 01.03.2021 